\title{
Radio imaging-spectroscopy observations of the Sun in decimetric and centimetric wavelengths
}

\author{
Yihua Yan ${ }^{1}$, Wei Wang ${ }^{1}$, Fei Liu ${ }^{1}$, Lihong Geng ${ }^{1}$, Zhijun Chen ${ }^{1}$, and \\ Jian Zhang ${ }^{2}$ \\ ${ }^{1}$ Key Laboratory of Solar Activity, National Astronomical Observatories, Chinese Academy of \\ Sciences, Beijing 100012, China. email: yyh@nao.cas.cn \\ ${ }^{2}$ Department of Astronomy, Peking University, Beijing 100871, China
}

\begin{abstract}
To address fundamental processes in the solar eruptive phenomena it is important to have imaging-spectroscopy over centimetric-decimetric wave range. The Chinese Spectral Radioheliograph (CSRH) in 0.4-15 GHz range with high time, space and frequency resolutions is being constructed to achieve this goal. The perspectives to open new observational windows on solar flares and CMEs will be achieved by mapping the radio emission from unstable electron populations during the basic processes of energy release. CSRH is located in a radio quiet region in Inner Mongolia of China. The array of CSRH-I in 0.4-2.0 GHz with $404.5 \mathrm{~m}$ antennas has been established and starts test observations. The $602 \mathrm{~m}$ antennas for array of CSRH-II in $2-15 \mathrm{GHz}$ have been mounted and assembled. The progress and current status of CSRH are introduced.
\end{abstract}

Keywords. Radioheliograph, soalr radio bursts, flares, CMEs, coronal magnetic field

\section{Motivation}

Radio bursts are prompt indicators of the various solar activities including flares and CMEs, etc. RHESSI observations indicate that non-thermal particles may account for a significant fraction of flare energy released during initial phase (Lin et al. 2008). Imaging spectroscopy over centimeter and decimetric wavelengths is important for addressing the problems of primary energy release, particle acceleration, and transportation processes (Bastian, et al. 1998, Gary \& Keller 2004, Aschwanden 2004, Pick \& Vilmer 2008)

From statistical study of radio dynamic spectral observations it was inferred that the acceleration site is located in a low-density region with a density of $n_{e}^{a c c} \sim 3 \times 10^{9}$ $\mathrm{cm}^{3}$, corresponding to a plasma frequency of $\nu_{p} \sim 500 \mathrm{MHz}$, from where electron beams are accelerated in upward (type III) and downward (RS bursts) directions (Aschwanden \& Benz 1997). However, the available radio imaging observations are presently only at a few discrete frequencies in the range 40 - $150 \mathrm{MHz}$ from Gauribidanur Radioheliograph (Ramesh et al. 1998), in the range 150 - $450 \mathrm{MHz}$ from Nancay Radioheliograph (Radioheliograph Group, 1989), at 5.7 GHz from Siberian Solar Radio Telescope (Grechnev et al. 2004), and at 17/34 GHz from Nobeyama Radioheliograph (Nakajima et al. 1994).

A flare event on 20 February 2002 at 11:07 UT was studied by Vilmer et al. (2002). They compared the hard X-ray images observed with RHESSI and of the radio emission sites observed by the NRH between 450 and $150 \mathrm{MHz}$. They found that variations in the HXR and decimetric emitting sources showed that both emissions were closely related to the energy release sites and to their variations during the flare. Thus they confirmed the previous suggestion of common acceleration/injection sites for HXR and 
Table 1. CSRH specifications

\begin{tabular}{|c|c|c|}
\hline Frequency range & & $400 \mathrm{MHz} \sim 15 \mathrm{GHz}(\lambda: 75 \sim 2 \mathrm{~cm})$ \\
\hline Frequency resoltuion & 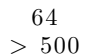 & $\begin{array}{l}\text { channels for CSRH-I in } 400 \mathrm{MHz} \sim 2 \mathrm{GHz} \\
\text { channels for CSRH-II in } 2 \sim 15 \mathrm{GHz}\end{array}$ \\
\hline Spatial resolution: & & I: $10^{\prime \prime} \sim 50^{\prime \prime}$ \\
\hline Temporal resolution: & & $\begin{array}{l}\text { II: } 1.3^{\prime \prime} \sim 10^{\prime \prime} \\
\text { I: } 25 \mathrm{~ms}\end{array}$ \\
\hline & & II: $\sim 200 \mathrm{~ms}$ \\
\hline Dynamic Range & & $25 \mathrm{db}$ (snapshot) \\
\hline Polarizations & & Dual circular L, R \\
\hline Array & & I: $40 \times \phi 4.5 \mathrm{~m}$ parabolic antennas \\
\hline & & II: $60 \times \phi 2 \mathrm{~m}$ parabolic antennas \\
\hline $\begin{array}{l}\text { Maximum baseline } \\
\text { Single dish beam (Field of view) }\end{array}$ & & $\begin{array}{c}3 \mathrm{~km} \\
0.6^{\circ} \sim 7^{\circ}\end{array}$ \\
\hline
\end{tabular}

decimetric/metric emitting electrons. However, as the lack of the knowledge of the magnetic connections between the emitting sites, it was not easy to understand in detail the link between the X-ray and radio sources and their evolution. So it is desired to image radio emissions from non-thermal electrons at even higher frequencies to better image the acceleration sites of the electrons which are responsible for HXR emission.

Trottet et al. (2006) carried out a detailed analysis of spectral and imaging observations of the November 5, 1998 flare obtained from hard X-rays and NRH radio images at 410, 327, 236 and $164 \mathrm{MHz}$. They found evidence that hard X-ray and decimetric/metric radio-emitting electrons were produced by the same accelerator. However, the available observations and analysis did not allow them to establish a clear and direct magnetic connection between the hard X-ray emitting region and the radio-emitting sources in the middle corona. Therefore the multi-frequency image observations in decimetric and centimetric wave range higher than $400 \mathrm{MHz}$ are needed.

For the famous December 13, 2006 flare event many radio fine structures were observed by Chinese Radiospectrometer at microwave range and it was found that the scale height ratio of plasma density to magnetic field deduced from the zebra patterns decreased by a factor of about 2 before and after the flare peak (Yan et al. 2007). The later case was further confirmed by statiscal resutls from 74 zebra pattern (Yu et al. 2012). However, what is the exact coronal magnetic field structure needs radio imaging-spectroscopy over wide frequency range in centimetric to decimetric wave range.

Therefore it is expected to have a new instrument that is capable of true imaging spectroscopy, with high temporal, spatial, and spectral resolution (Hudson \& Vilmer 2007, Pick \& Vilmer 2008). The Chinese Spectral Radioheliograph (CSRH, Yan et al. 2004) and Frequency-Agile Solar Radiotelescope (FASR, Bastian 2003, Gary 2003) have been proposed to reach this goal.

The project of the Chinese Spectral Radioheliograph (CSRH) has been supported as National Major Scientific Research Facility Program of China and is under construction now, which will open new observational windows on flares and CMEs at radio wavelengths. The whole budget for the instruments of CSRH project was approved in 2009 . The site survey for the CSRH array was completed at Mingantu town in Inner Mongolia of China and the radio quiet zone protection with $10 \mathrm{~km}$ radius centered at CSRH site was setup in 2008. The construction also started in the autumn of 2008. The CSRH project is due to finish in 2013.

\section{Description of CSRH}

The Chinese Spectral Radioheliograph (CSRH) in centimetric-decimetric wave range is a solar-dedicated radio interferometric array that will be used to carry out imaging 
Table 2. Current measured CSRH-I antenna locations in local coordinates (Unit: $\mathrm{m}$ )

\begin{tabular}{crrrrrrrrrrr}
\hline Arm A & $\mathrm{X}$ & $\mathrm{Y}$ & $\mathrm{Z}$ & $\operatorname{Arm~B}$ & $\mathrm{X}$ & $\mathrm{Y}$ & $\mathrm{Z}$ & $\operatorname{Arm~C}$ & $\mathrm{X}$ & $\mathrm{Y}$ & $\mathrm{Z}$ \\
IA0 & 0 & 0 & 0 & & & & & & & & \\
IA1 & -0.022 & 7.988 & 0 & IB1 & -6.884 & -4.112 & 0 & IC1 & 6.966 & -3.990 & 0 \\
IA2 & -6.426 & 19.456 & 0 & IB2 & -13.559 & -15.394 & 0 & IC2 & 20.185 & -4.093 & 0 \\
IA3 & -21.025 & 28.661 & 0 & IB3 & -14.214 & -32.499 & 0 & IC3 & 35.379 & 3.976 & 0 \\
IA4 & -44.371 & 31.799 & 0 & IB4 & -5.365 & -54.364 & 0 & IC4 & 50.088 & 26.883 & 0 \\
IA5 & -79.806 & -0.535 & 0.1 & IB5 & 40.334 & -68.776 & 0 & IC5 & 39.461 & 69.250 & 0 \\
IA6 & -92.243 & -67.981 & 0 & IB6 & 105.005 & -45.994 & 0 & IC6 & -12.702 & 113.930 & 0 \\
IA7 & -49.823 & -156.924 & 0 & IB7 & 160.745 & 35.210 & 0 & IC7 & -110.987 & 121.621 & 2 \\
IA8 & 75.111 & -226.202 & 0 & IB8 & 158.353 & 178.005 & 0 & IC 8 & -233.431 & 47.856 & 2 \\
IA9 & 283.605 & -203.226 & 0 & IB9 & 34.311 & 347.220 & 0 & IC9 & -317.836 & -143.928 & 2 \\
IA10 & 517.111 & 3.454 & 0 & IB10 & -261.386 & 446.416 & 5.5 & IC10 & -255.740 & -449.563 & 2 \\
IA11 & 624.882 & 459.903 & 0 & IB11 & -710.941 & 311.107 & 10 & IC11 & 86.093 & -771.081 & 0 \\
IA12 & 356.229 & 1120.404 & 5.5 & IB12 & -1148.753 & -251.691 & 10 & IC12 & 792.346 & -868.716 & 10 \\
IA13 & -286.460 & 1817.530 & 10 & IB13 & -1194.157 & -1342.201 & 10 & IC13 & 1759.184 & -362.106 & -0.1 \\
\hline
\end{tabular}

Central antenna IA0 location: E $115^{\circ} 15^{\prime} 1.8^{\prime \prime}$, N $42^{\circ} 12^{\prime} 42.6^{\prime \prime}$, Altitude $1365 \mathrm{~m}$

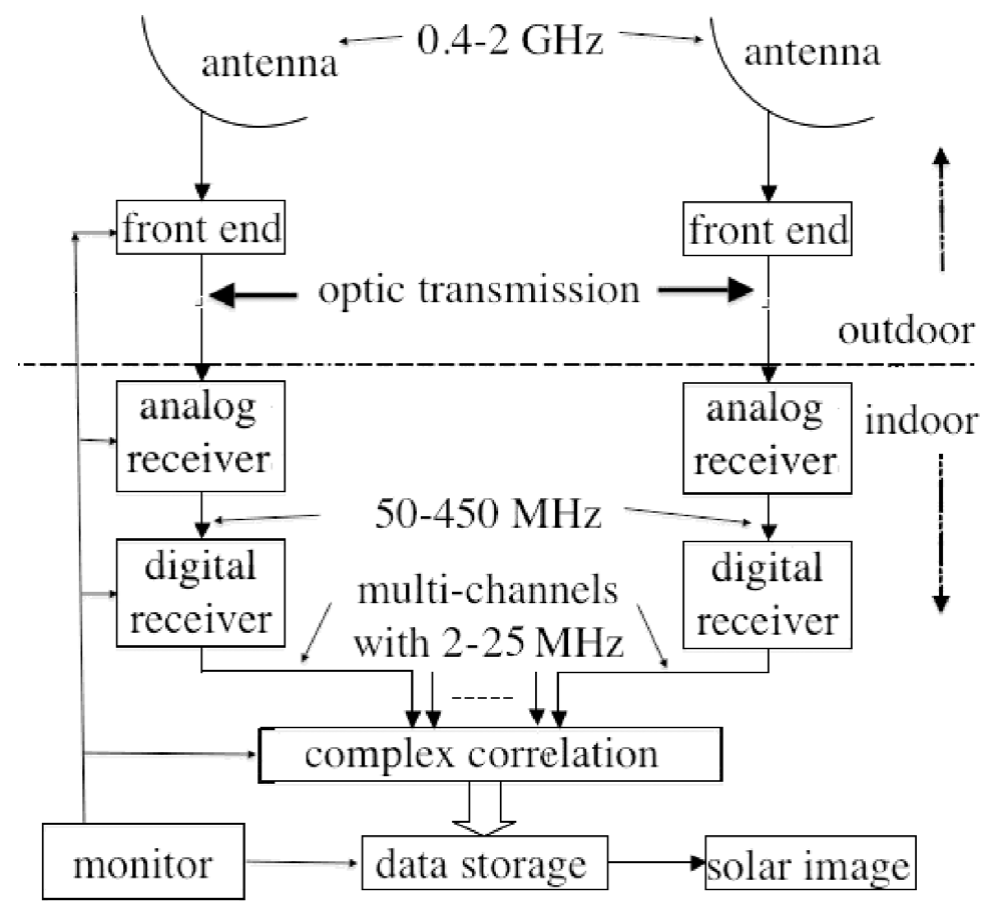

Figure 1. The system block diagram of CSRH-I. CSRH-II has a similar system structure except that the $\mathrm{RF}$ input is $2-15 \mathrm{GHz}$ in place of $0.4-2 \mathrm{GHz}$.

spectroscopy of the Sun, to produce high spatial resolution, high time resolution and high frequency resolution images of the Sun simultaneously (Yan et al. 2004, Yan et al. 2009). The updated main specifications of CSRH are listed in Table 1.

The CSRH frequency range is $0.4-15 \mathrm{GHz}$ which is divided into CSRH-I covering 0.4$2 \mathrm{GHz}$ band and CSRH-II covering $2-15 \mathrm{GHz}$ band. The CSRH-I contains 40 antennas of $4.5 \mathrm{~m}$ diameter, and the CSRH-II contains 60 antennas of $2 \mathrm{~m}$ diameter. The complete system of CSRH-I array has been established and is in test observation now, whereas 60 antennas of CSRH-II have just been mounted in the field in 2012 and the whole system of CSRH-II is due to finish in 2013. The whole 100 antennas of both CSRH arrays are located 
on 3 log-spiral arms, and the maximum baseline length is $3 \mathrm{~km}$. The currently measured antenna locations for CSRH-I array is listed in Table 2. The system block diagram for CSRH-I in dm-wave range is shown in Figure 1. CSRH-II system has a similar structure as in Figure 1 except that the RF input is $2-15 \mathrm{GHz}$ instead of $0.4-2 \mathrm{GHz}$. Additionally two $20 \mathrm{~m}$ antennas for interferometry experimental in 400-1000 MHz were established in 2011 in CSRH site, which may be incorporated into CSRH-I calibrations later.

From Figure 1 it is seen that the solar radio emission in $0.4-2 \mathrm{GHz}$ is detected by each CSRH-I antenna with the broadband feed and outdoor devices including LNA and optic transmitter. The signal with $400 \mathrm{MHz}$ bandwidth, which covers the whole 1.6 $\mathrm{GHz}$ bandwidth by scanning 4 times, is then transmitted through optic fibers to indoor devices including optic receiver and analogous receivers with an output in $50-450 \mathrm{MHz}$ range. It is then followed by digital receiver with 1 Gsps A/D converter to receive 400 $\mathrm{MHz}$ analogous signal and the digital receiver outputs 16 channels simultaneously for the complex correlations with $\sim 2-25 \mathrm{MHz}$ bandwidth for each channel. The time delay compensation and fringe stopping are considered in the digital correlations. The whole correlation procedure is controlled by a monitoring subsystem. Similar procedure applies to CSRH-II except that the solar radio emission in $2-15 \mathrm{GHz}$ is detected by each CSRH-II antenna with the broadband feed and the IF signal with $400 \mathrm{MHz}$ bandwidth will need to scan the whole $13 \mathrm{GHz}$ bandwidth by 33 times now.

\section{Progress of CSRH Construction}

Among the technical issues for CSRH system based on aperture synthesis technique (Taylor et al. 1999, Thompson et al. 2001), a key problem is that the high performance ultra-wide band feed is needed for reflector antennas. Such feed should be with wide impedance bandwidth, low profile, symmetrical radiation patterns, and fixed phase center over whole band. The Eleven feed (Olsson et.al. 2006) has many advantages and is a good option. For its original version the return loss is less than $-5 \mathrm{~dB}$ (or VSWR $\leqslant$ 3.53), which satisfies the requirements for observing other astrophysical objects. However, solar radio bursts and accordingly their polarizations may vary quickly. Therefore, the isolation is important for correct observations of the polarizations. So the return loss or VSWR should be further reduced in order to achieve this goal. We have successfully developed the feeds for CSRH-I with VSWR less than 1.5 over most the frequency range and exhibits low profile, $133 \%$ impedance bandwidth and good radiation characteristics. Figure 2 shows the polarization degree measurement of IC8 antenna element in $400 \mathrm{MHz}$ $-2 \mathrm{GHz}$ range. It is seen that in nearly $90 \%$ frequency band the polarization degree, or $\left(P_{L}-P_{R}\right) /\left(P_{L}+P_{R}\right)$ is less then $5 \%$, which indicates good isolation performance.

As mentioned above, CSRH-I has been put into test observation and fixed time delays among CSRH-I array have been measured for calibration. The measured RMS variations are $<1$ ns which is very robust, as similar results obtained after multiple measurements pursued 1 years apart (Liu et al. 2012). For the test observations, fringes of the satellite signals, the Sun, and Cygnus A have been obtained correctly for all baselines. The residuals of the phase closures for every tri-antenna composition were about 2 degrees for both geostationary and GPS satellites. Fringe stopping has been obtained for short and mid baselines when observing the quiet Sun. These experiments verify the system design and demonstrate the system performance. The two $20 \mathrm{~m}$ antennas in 400$1000 \mathrm{MHz}$ will be incorporated into CSRH-I calibrations. It is expected to obtain CSRH-I first solar maps soon.

In October 2012, 60 CSRH-II antennas of 2m diameter were erected at the CSRH site whereas the analogous and digital receivers, digital correlators, and monitor subsystem, 


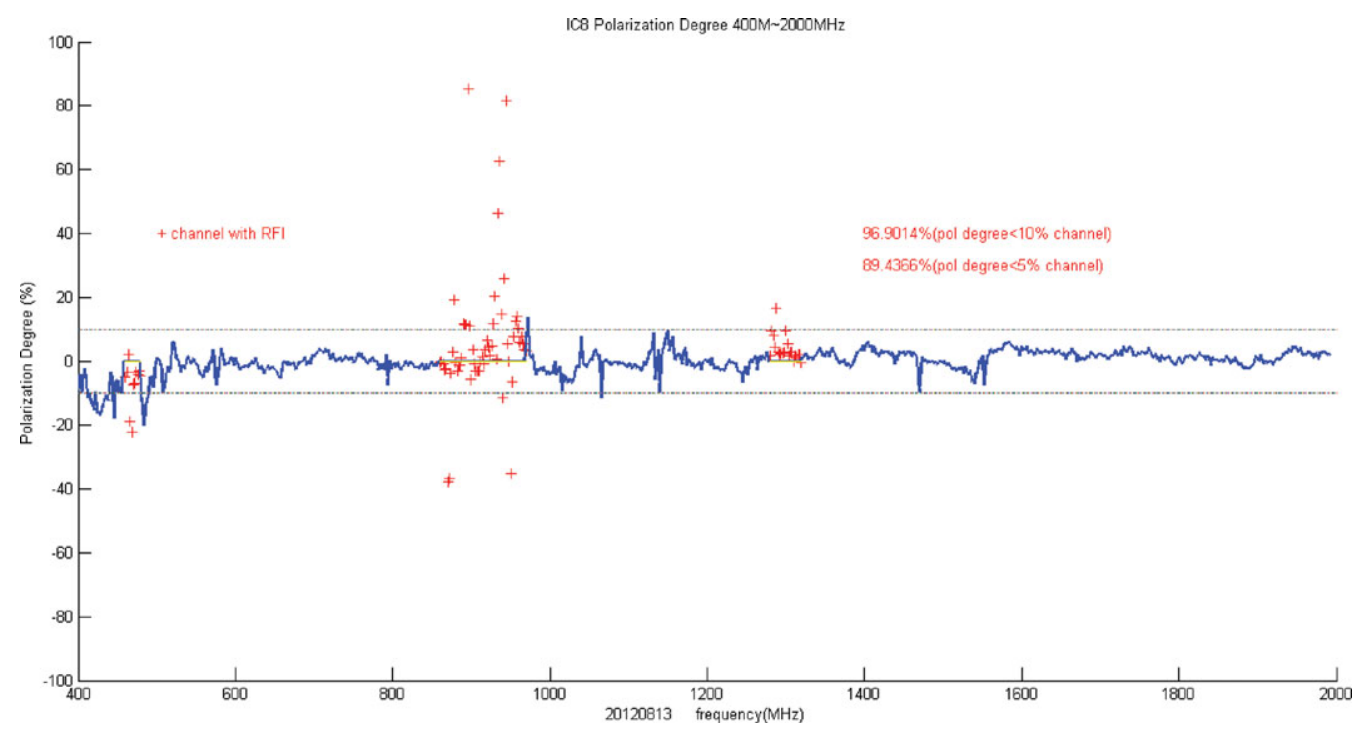

Figure 2. Polarization degree, or $\left(P_{L}-P_{R}\right) /\left(P_{L}+P_{R}\right)$ of a CSRH-I antenna element in 400 $2000 \mathrm{MHz}$ range measured on $13 \mathrm{Aug} 2012$, which is $\leqslant 10 \%$ over $96.9 \%$ whole frequency band, and furthermor, $\leqslant 5 \%$ over $89.4 \%$ whole frequency band. The red plus sign indicates frequency point where strong RFI occurred.

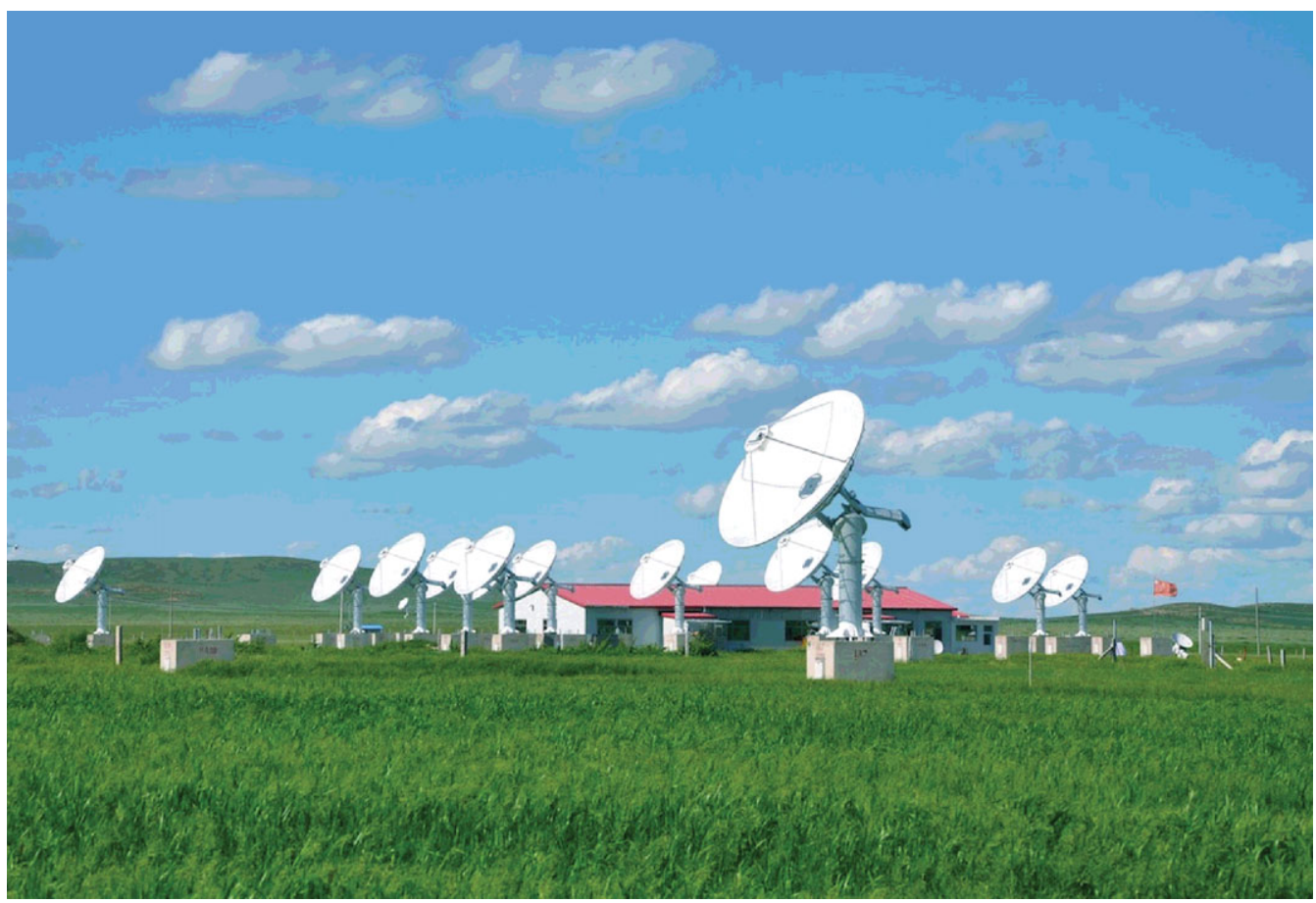

Figure 3. Central part antennas of CSRH-I array for test observation on 8 August 2012.

etc. are still under development and manusfactures in laboratories and due to assemble and test in 2013. Figure 3 shows the central part antennas of CSRH-I array for test observation on 8 August 2012. 


\section{Conclusion}

In summary, radio imaging spectroscopy is in its infancy and will open new observational windows on flares and CMEs, it will also provide coronal magnetograms. To reach this goal, CSRH project has been funded and will be fulfilled completely by the end of 2013. The radio quiet zone protection with $10 \mathrm{~km}$ radius for CSRH was established. The CSRH-I array in decimetric wave range has been established during 2008-2011. For the test observations, the phase fringes for all baselines have been obtained. The fringe stopping was obtained for short and mid baselines of the quiet Sun. It is still for calibration and verification now and it is expected to obtain first solar maps soon. The all 60 antennas for CSRH-II array have been mounted and the analogous and digital receivers, digital correlators, and monitor subsystem for CSRH-II will be assembled in 2013.

\section{Acknowledgement}

The CSRH is supported by National Major Scientific Research Facility Program of China with the grant Number ZDYZ2009-3. The NFSC Grants Nos.11221063, 10921303 and MOST Grant No.2011CB811401 are acknowledged.

\section{References}

Aschwanden, M. J. \& Benz, A. O. 1997, ApJ, 480, 825

Aschwanden, M. J. 2005, Physics of the Solar Corona, Springer, Berlin, 2005.

Bastian, T. S., Benz, A. O., \& Gary, D. E. 1998, ARAA, 36, 131

Bastian, T. S. 2003, Proc. SPIE, 4853, 98

Gary, D. E. 2003, J KAS, 36, 135

Gary, D. E. \& Keller, C. U. 2004, Solar and space weather radiophysics, Kluwer, Dordrecht, 2004 Grechnev, V. V., Lesovoi, S. V., Smolkov, G. Ya., et al. 2003, SolPhys, 216, 239

Hudson, H. \& Vilmer, N. 2007, Lect Notes Phys, 725, 81

Nakajima, H., Nishio, M., Enome, S., et al. 1994, Proc. IEEE, 82, 705

Olsson, R., Kildal, P. S., \& Weinreb, S. 2006, IEEE Trans Ant Prop, 54, 368

Pick, Monique \& Vilmer, Nicole 2008, Astron Astrophys Rev, 16, 1

Radioheliograph Group 1989, SolPhys, 120, 193

Ramesh, R., Subramanian, K. R., Sundararajan, M. S., \& Sastry, Ch. V. 1998, SolPhys, 181, 439

Thompson, A. R., Moran, J. M., \& Swenson, G. W. Jr. 2001, Interferometry and Synthesis in Radio Astronomy, John Wiley \& Sons, New York, 2001

Taylor, G. B., Carilli, C. L., \& Perley, R. A. Synthesis Imaging In Radio Astronomy, II, 1999

Trottet, G., Correia, E., Karlick, M., Aulanier, G., Yan, Y., \& Kaufmann, P. 2006, SolPhys, 236, 75

Vilmer, N., Krucker, S., \& Lin, R. P., Rhessi Team 2002, SolPhys, 210, 261

Yan, Y. H., Zhang, J., Wang, W., Liu, F., Chen, Z. J., \& Ji, G. S.2009, Earth Moon Planet, 104, 97

Yan, Yihua, Huang, Jing, Chen, Bin, \& Sakurai, Takashi 2007, PASJ, 59, 815

Yan, Y. H., Zhang, J., Huang, G., et al. 2004, Proc. 2004 Asia-Pacific Radio Science Conference, Qingdao, China, IEEE, Beijing, p. 391

Yu, Sijie, Yan, Yihua, \& Tan, Baolin 2012, ApJ, 761, 136 